\title{
Concept and Realisation of an implantable EMG and motion measuring device
}

\author{
Stefan Weber Member IEEE, Tim C. Lueth, Member IEEE \\ Central Institute for Medical Technology • Technical University Munich \\ Boltzmannstrasse 11, Garching, Germany
}

\begin{abstract}
In this contribution we are reporting on the concept and development of an integrated and implantable device for EMG measurement inside of human bodies. Such devices are capable of measuring EMG potentials of muscle activity during dedicated activities and of transmitting such information to the outside of the body. They will serve for extended possibilities of human machine interaction in the future. The wearer will be able to control devices and systems apart from his body by simple muscle activity. Such devices have to be within acceptable technological limits - size, weight - to become accepted. In this contribution we are demonstrating the concept and give insight into technological aspects of the developed systems and present first results on the analysis of the available data.
\end{abstract}

Keywords: EMG measurement, motion sensing and analysis implant;

\section{INTRODUCTION}

In this article we are describing first results in developing a novel type of implantable EMG sensor that will be used by humans in the future to control devices and systems that are apart from the human body. Such a device is an interesting option to existing types of human-machine interfaces. One can imagine, that simply moving an arm and or waving the hand to turn the room light on or controlling household devices and artificial limbs by gesture forming becomes an immediate option.

We believe that as soon as such sensor devices will be small and independent enough to be easily implanted, the given approach will become interesting. In the following article we will introduce the concept of a MART - Muscle Activity Recorder and Transmitter, the system setup and first experiments being conducted to demonstrate a general feasibility. In the following subchapter a general state of the art in implantable technology is described and critically analyzed.

\section{STATE OF THE ART}

\section{A. Electromyography}

Electromyography (EMG) is the measurement of electrical activity of a set of muscles. Electromyographic potentials are measured by using electrodes, that are placed on the skin (Surface EMG) or needle electrodes that are directly placed within the muscle tissue. Clinically, through interpretation of derived electrical potentials impairment of muscles and lesions can be identified. Indications for an EMG measurement are a) differentiation of muscle impairment; b) muscle weakness and movement dysfunction with/without Athrophy; c) neuromuscular transmission problems. Additionally, EMG potentials are also used for activity analysis for mobile patients [1].

Intramuscular EMG measurements are a precise method to to determine muscle contractions and to derive information about gestures, activities and motion pattern. The availability of the introduced implantable EMG measurement system is becoming interesting to determine exact commands to control an artificial prosthesis. On the other hand, such a device can become a novel form of interface between human beings and machines as well as computers, where applying certain motion patterns triggers a device to start a desired action.

\section{B. Functional implantable devices}

The current scientific and experimental state of the art has to cite a great number of approaches and (pre)clinical applications with regard to functional implantable devices. Functional refers to the capability of an implant to incorporate subsystems and components to measure, analyse biological signals, triggers actions and communicate measurement results to the outside of the body or to different and distant parts of the body. Functional implants are nowadays capable to measure

- Cardiac parameters such as ECG signals, consistency of electrolytes and muscle elasticity [2];

- Gastroesophageus parameters such as pressure, $\mathrm{pH}$ values and glucose level [3];

- Vascular flow information such as peak flow, velocity and volume flow [4];

- Osseous parameters such elongation and healing state and process [5];

- Neurological parameters such as the pressure within th inner eye [6] and the brain [7];

First promising results have also been achieved in the area of artificial retina implants [8]. However, in daily clinical routine, today only cardiac pacemakers and Chochlear Implants (CI) are available.

\section{Wireless protocolls}

A number of wireless protocols which are designed for certain purposes, exist today. The main differentiation criteria are available bandwidth and distance. Here the Bluetooth and 
Zigbee standard focus on short range and considerable low to medium bandwidth. Additionally, a variety of proprietary protocols exist, that provide a single chip hardware interface that is capable of being integrated in smallest applications (ANT, Chipcon).

\section{Motion and acceleration measurement}

The measurement of motion and acceleration is an often occurring challenge in technical and biological processes. In a physical sense, motion is always connected to the appearance of other physical parameters such as force, velocity and distance. Other application areas include automobile technology, robotics, in the air and space industry, but also in medicine and sports. Measurement tasks include detection of inclination, vibrations and physical shock.

Additionally, acceleration data is combined with other sensor readings to support navigation applications, motion analysis and medical related diagnostic challenges. To technically measure acceleration different principles are available that rely on a number of applicable physical effects [9]:

$$
\begin{aligned}
& \text { - piezo-electric; } \\
& \text { - } \quad \text { piezo-resistive; } \\
& \text { - } \quad \text { capacitive; } \\
& \text { - magnetical; } \\
& \text { - inductive; } \\
& \text { - } \quad \text { optical; } \\
& \text { - } \quad \text { thermal. }
\end{aligned}
$$

The possibilities in silicium technology currently allow the realisation of micro-electro-mechanical systems based on piezo-electric, piezo-resistive and capacitive effects.

\section{MATERIAL AND METHODS}

\section{A. Static system description}

The implant was realized battery powered device. Recharging of the device is achieved by means of an inductive coupling. The electrical energy is induced through a set of two coils, the primary coil placed outside the body and the secondary coil integrated within the housing of the implant (Figure 1).

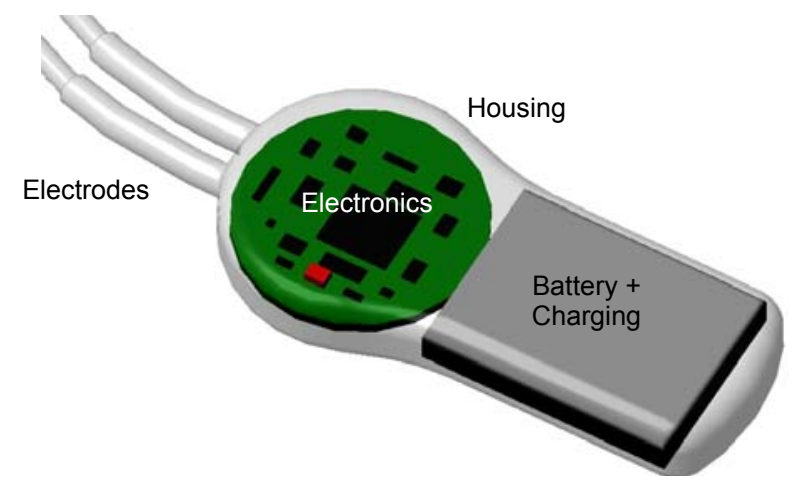

Figure 1. Design of the implant prototype showing the different system components.
The device is controlled by a ATmega128L micro processor. For communication, a Chipcon CC1100 provides a wireless interface at $916 \mathrm{MHz}$. All electronics are integrated within a central electronics unit (MICA2dot, Crossbow). Within a separate measurement modul, operational amplifiers for the amplification of the EMG signals and a 3 DoF acceleration sensor are integrated and connected to the central unit. As electrodes, a uniploar and a bipolar pacemaker electrodes are integrated. The central unit is connected to the electrodes (Medtronic). Additionally, a Lithium-Polymer rechargeable battery is connected and integrated.

The container of the implant consists of a biocompatible silicon (PolyTec/NuSil) that can be implanted up to 30 days. For integration of the electronics into the housing container, a molding form was manufactured and the electronics are placed inside the form. After the form is closed, the silicon is injected into the form. To remove enclosed air from the form, it is placed in a vacuum chamber and vacuum is applied for a short time. After polymerisation the form is removed and the implant can be removed.

\section{B. Signal analysis and Processing}

The sensed muscle potentials are amplified by the operation amplifiers $\left(\mathrm{k}_{\text {amp. }}=1000\right)$ and are $\mathrm{A} / \mathrm{D}$ converted by the controller. Signals of acceleration sensor are also read by the micro controller with 10 bit resolution. Data is low pass filtered and then transmitted wirelessly to a base station for further processing. The base station consists of a receiver with a connected personal computer for further processing of the data.

Technically, the implants micro controller is capable of providing sufficient resources for processing and analysing EMG and acceleration data, which will be integrated in the future. For the time, the transmission and analysis of raw data is required to conduct the necessary experiments.

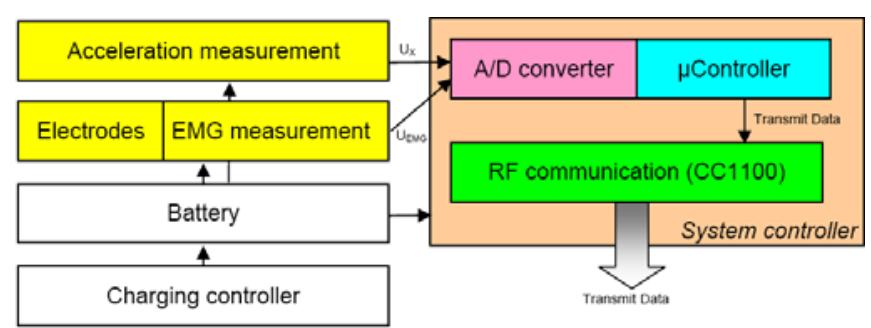

Figure 2. System setup and component of the EMG and motion sensing implant.

\section{Experimental setup}

To demonstrate the functionality of the implant, a first experiment was conducted. The motivation of the experiment is to analyse the available sensor readings after their transmission, reduction and broadcasting over the wireless connection. For this experiment, an implant was produced and integrated (see Figure 3). For the experiment, two different measurement modalities were taken into account, one being the implant measurement and the second a oscilloscope measurement.

The electrodes were attached to a test person's upper arm and EMG signals were recorded subsequently. After the preprocessing and transmission of the data, the recordings were 
analyzed. In parallel, oscilloscope probes were connected to the electrodes to analyse the resulting EMG potential in real time. In a later analysing phase the two sensor readings were compared and analysed (see Figure 4 and 5).

\section{RESUlTS}

A first prototype of the implant was fabricated with the exact spatial dimensions and the desired functionalities. The electronic components were realized using foil based conductor boards. The dimensions of all components were kept small adequately, to allow for an optimal integration into the implant housing.

The implant housing consists of biocompatible silicon with attached silicon electrodes. The diameter of the implant is 23 $\mathrm{mm}$ with a thickness of $8 \mathrm{~mm}$. The length of the electrodes is $60 \mathrm{~cm}$ (typical length of pacemaker electrode). The length of the electrode will allow for distant implantation of the actual implant away from the point of measurement.

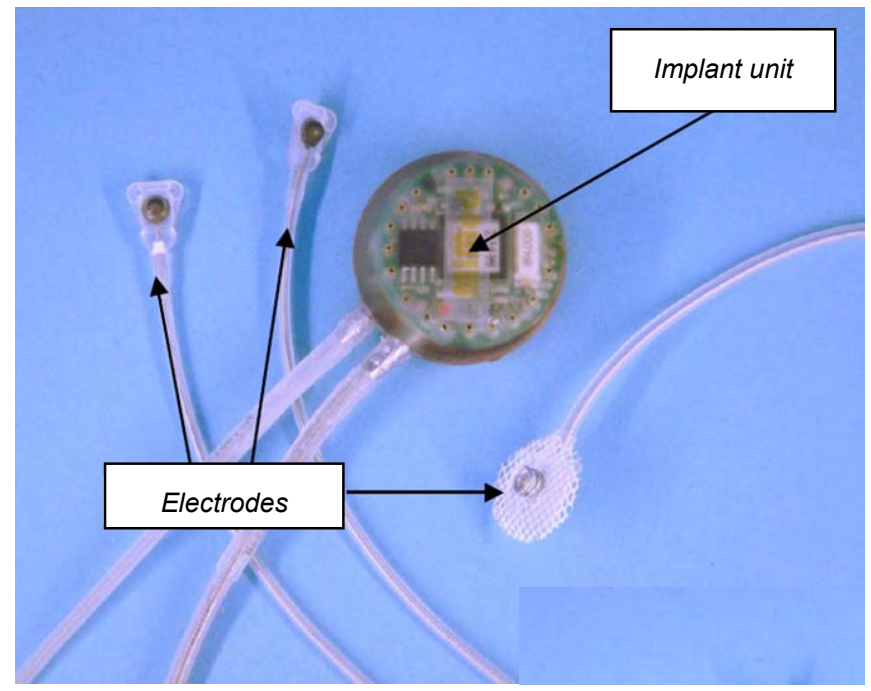

Figure 3. Molded and integrated implant system with electrodes.

\section{A. Analysis of arm motion activity}

For the first validation, the measurement of the contractions of the bizeps on the upper arm was conducted. The EMG signals were recorded using an oscilloscope (Figure 4) and using the base station of the implant system (Figure 5).

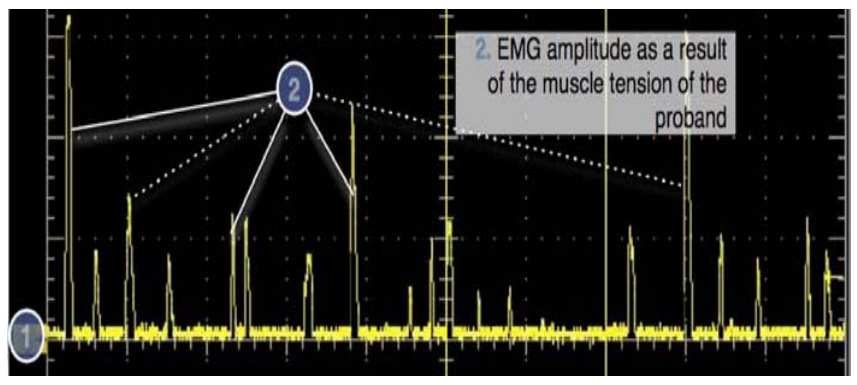

Figure 4. Depiction of EMG data, recorded directly with an oscilloscope (positive signal amplification).
The most significant result of the experiement was the determination of the actual time delay of approximately 2 seconds. The delays integrate over the additional processing and transmission steps. The two measurement modalities are compared qualtitatively and contractions of the muscles are well represented in the implant sensor reading.

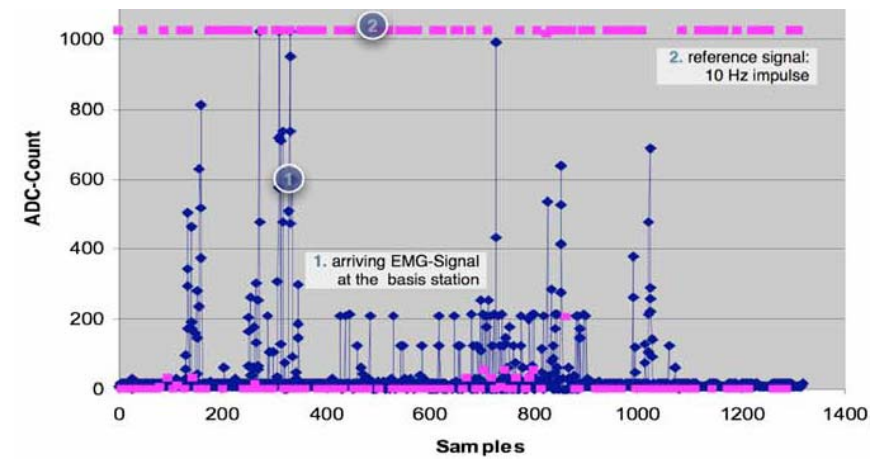

Figure 5. Analysis of recorded EMG measurements after transmission from the implant. A typical time delay of two seconds was experienced in the current setup.

\section{DISCUSSION}

The design and realisation of novel implant technology for scientific purposes and low volume systems is feasible today. With respect to available micro computer technology, sensor components (especially electrodes) from implant manufacturers and the wireless technology the realisation of such devices is worthwhile. Another very promising option for manufacturing implant housings for first experimental validation processes is the application of polymer bases silicon. It enables for fast and precise production of simple, yet useful and applicable housings. A drawback of silicon housings is the missing impermeability against body fluids over a longer period of time. Such silicon based implants are currently not well suited for long time application.

Next steps include the manufacturing of a set of implants and the preparation of an animal testing phase. We aim, to implant the introduced implants into pigs to evaluate the possibilities in connecting the electrodes to the muscle, optimal places for implantations and the evaluation of a stable method for transmitting data from within the body.

\section{ACKNOWLEDGMENT}

The authors would like to thank Dipl.-Ing. Stefan Mayer and Christian Reisert (M.Sc.) for conducting the integration work and carrying out the necessary experiments.

\section{REFERENCES}

[1] Honda Y., Weber S., Lüth T. C. (2006): Funkbasierte EMG-Messung für die Echtzeit-Auswertung von Laufaktivität. VDE Workshop Biosignalverarbeitung, Potsdam, 13. - 14. Juli 2006. Potsdam Juni 2006

[2] Manoli, Y. (1998): Chips, die einem am Herzen liegen. Magazin Forschung 1/1998, Universität des Saarlandes

[3] Valdastri, P. et al. (2004): An Im-plantable Telemetry Platform System for In Vivo Monitoring of Physiological Parameters. IEEE Transactions of Information Technology in Biomedicine, Vol. 8, No. 3, September 2004. 
[4] Zacheja, J., Bach, T. (2002): A Telemetric Measurement System for Flow Diagnostic after Bypass Surgery. Biomed Tech (Berlin), 2002.

[5] Rieger, P. (2001): Implantierbare Meßsysteme mit drahtloser digitaler Informationsübertragung. Dissertation im Fachbereich Elektrotechnik der Universität Kaiserslautern, 2001.

[6] Draeger, J. (2002): Intraokulares Implantat zur Messung des intraokularen Druckes. Eu-ropäische Patentanmeldung, EP 1232723 A1, 2002.
[7] Stieglitz, T. et al. (2005): Implant-able Biomedical Microsystems for Neural Prostheses - Flexible, Polyimide-Based, and Modular. IEEE Engineering in Medicine and Biology Magazine, September 2005.

[8] Kolnsberg, S. (2001): Drahtlose Signal- und Energieübertragung mit Hilfe von Hochfrequenztechnik in CMOS-Sensorsystemen. Dissertation im Fachbereich Elektrotechnik der Gerhard-Mercator-UniversitätGesamthochschule Duisburg, 2001.

[9] Tränkler, HR., Obermeier, E.: Sensortechnik - Handbuch für Praxis und Wissenschaft. Springer Verlag, Berlin, Heidelberg, New York, 1998. 